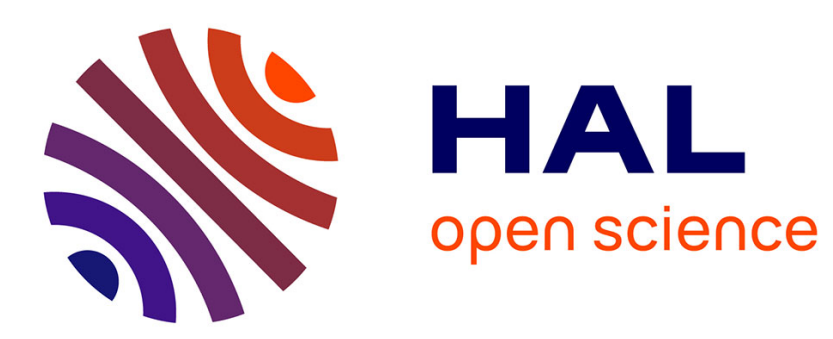

\title{
How many surface plasmons are locally excited on the ridges of metallic lamellar gratings?
}

Bing Wang, Philippe Lalanne

\section{To cite this version:}

Bing Wang, Philippe Lalanne. How many surface plasmons are locally excited on the ridges of metallic lamellar gratings?. Applied Physics Letters, 2010, 96, pp.051115. 10.1063/1.3304021 . hal-00570644

\section{HAL Id: hal-00570644 \\ https://hal-iogs.archives-ouvertes.fr/hal-00570644}

Submitted on 5 Apr 2012

HAL is a multi-disciplinary open access archive for the deposit and dissemination of scientific research documents, whether they are published or not. The documents may come from teaching and research institutions in France or abroad, or from public or private research centers.
L'archive ouverte pluridisciplinaire HAL, est destinée au dépôt et à la diffusion de documents scientifiques de niveau recherche, publiés ou non, émanant des établissements d'enseignement et de recherche français ou étrangers, des laboratoires publics ou privés. 


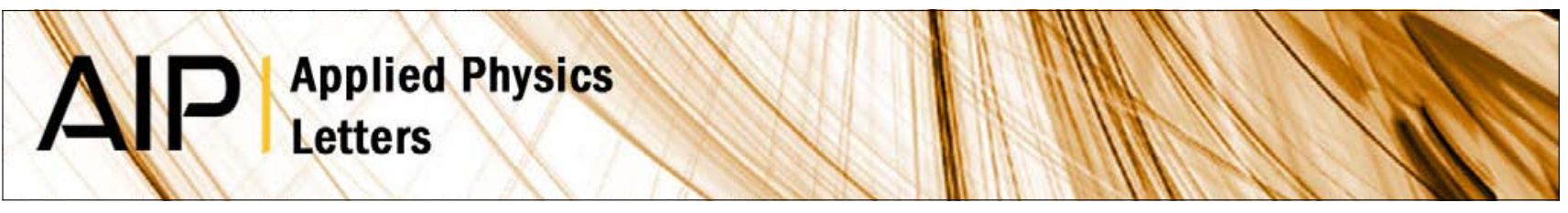

How many surface plasmons are locally excited on the ridges of metallic lamellar gratings?

B. Wang and P. Lalanne

Citation: Appl. Phys. Lett. 96, 051115 (2010); doi: 10.1063/1.3304021

View online: http://dx.doi.org/10.1063/1.3304021

View Table of Contents: http://apl.aip.org/resource/1/APPLAB/v96/i5

Published by the American Institute of Physics.

Additional information on Appl. Phys. Lett.

Journal Homepage: http://apl.aip.org/

Journal Information: http://apl.aip.org/about/about_the_journal

Top downloads: http://apl.aip.org/features/most_downloaded

Information for Authors: http://apl.aip.org/authors

\section{ADVERTISEMENT}

iPeerReview

AIP's Newest App

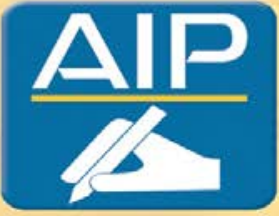

Authors...

Reviewers...

Check the status of

submitted papers remotely!

AIP Publishing 


\title{
How many surface plasmons are locally excited on the ridges of metallic lamellar gratings?
}

\author{
B. Wang ${ }^{a}$ and P. Lalanne \\ Laboratoire Charles Fabry de l'Institut d'Optique, CNRS, Université Paris-Sud, Campus Polytechnique, RD \\ 128, 91127 Palaiseau Cedex, France
}

(Received 8 November 2009; accepted 11 January 2010; published online 3 February 2010)

\begin{abstract}
In contrast to earlier classical studies that analyze the surface Bloch modes supported by metallic gratings composed of slits as collective surface-plasmon-polaritons (SPPs) resonances (or poles) of the entire periodic problem, we study the normalized rate of SPPs that are locally launched on every individual ridge of metallic lamellar gratings. With this "microscopic" description at the unit-cell level, we further explain how these individual SPPs constructively interfere to build up the classical collective resonances. The approach, which combines analytical treatments and fully-vectorial computations, shines new light on an important and classical phenomenon of grating diffraction.

(C) 2010 American Institute of Physics. [doi:10.1063/1.3304021]
\end{abstract}

Since Wood's discovery ${ }^{1}$ of resonance anomalies in the spectrum of light resolved by optical diffraction gratings, metal micro and nanostructured surfaces have received considerable attention ${ }^{2}$ and are nowadays at the heart of various scientific and technological challenges. ${ }^{3}$ The interest comes from the rich optical response of these surfaces that may support resonance states. The latter are intrinsically due to elementary multiple scatterings that occur at the unit-cell level between two different kinds of individual surface waves, the surface plasmon polaritons (SPPs) ${ }^{2,3}$ and the quasi-cylindrical waves (quasi-CW), which has been studied in the recent literature, see Refs. 4-6 for a review of their optical properties for two-dimensional indentations. At visible frequencies, because they are well confined on noble metal interfaces, SPPs are efficiently excited by subwavelength indentations ${ }^{6}$ and strongly involved into the rich physics of nanostructured surfaces. However, despite significant efforts provided for analysis the scattering of SPPs by individual indentations, ${ }^{7-10}$ by slit doublets ${ }^{11-13}$ or by more complicated systems, ${ }^{14}$ there is no comprehensive picture that explains how the collective optical responses build up from the individual SPPs scattered by the indentations. Presently, one even ignores how many SPPs are excited on the flat parts of the nanostructured surface in between the indentations, even for basic metallodielectric geometries such as slit arrays.

In this letter, we study the local excitation of SPPs that are launched on the flat ridge interfaces of lamellar gratings (Fig. 1). Through rigorous full-vectorial calculations and approximate closed-form expressions, we surprisingly find that the normalized SPP-excitation rate is independent of the metal conductivity. This result, which strongly contrasts with previous studies on isolated apertures, ${ }^{4-6,10}$ is further explained with a microscopic model that involves elastic and inelastic multiple scatterings at every individual slit.

The lamellar geometry considered in this work is shown in Fig. 1(a). It is composed of an array (periodicity $a$ in the $x$-direction) of semi-infinite slits (width $w$ ) perforated in a

\footnotetext{
${ }^{a}$ Present address: Institute of Materials Research and Engineering, 3 Research Link, Singapore 117602. Electronic mail: wangb@imre.astar.edu.sg.
}

gold substrate. The grating is surrounded by a dielectric half-space (relative permittivity $\varepsilon_{\mathrm{d}}$ ) and the dispersive permittivity $\varepsilon_{\mathrm{m}}$ of gold is taken from Ref. 15 . The grating is illuminated by a plane wave $\left(k_{0}=2 \pi / \lambda=\omega / \mathrm{c}\right)$ with a unitary magnetic field parallel to the slits. We denote the transverse scattered field by $\left[\mathrm{H}_{\mathrm{y}}(x, z), \mathrm{E}_{\mathrm{z}}(x, z)\right]$ and the transverse fields of a normalized SPP mode of a flat interface by $\left[\mathrm{H}_{\mathrm{y}, \mathrm{SP}}(z), \mathrm{E}_{\mathrm{z}, \mathrm{SP}}(z)\right] \exp \left(\mathrm{jk}_{\mathrm{SP}} x\right)=\left[1, k_{\mathrm{SP}} /\left(\kappa \omega \varepsilon_{0}\right)\right] \exp \left(\mathrm{i} \gamma_{\mathrm{SP}} z\right)$ $\times \exp \left(\mathrm{jk}_{\mathrm{SP}} x\right)$, with $\kappa=\varepsilon_{\mathrm{d}}$ or $\varepsilon_{\mathrm{m}}$ for $z>0$ or $z<0, \gamma_{\mathrm{SP}}=\left(\kappa k_{0}^{2}\right.$ $\left.-k_{\mathrm{SP}}^{2}\right)^{1 / 2}$ and $k_{\mathrm{SP}}=k_{0}\left[\varepsilon_{\mathrm{d}} \varepsilon_{\mathrm{m}} /\left(\varepsilon_{\mathrm{d}}+\varepsilon_{\mathrm{m}}\right)\right]^{1 / 2}$. By applying the orthogonality theorem of normal modes for lossy systems, the SPP-excitation rates on the ridges between the slits can be written as ${ }^{6}$

$$
\begin{aligned}
e_{\mathrm{SP}}^{ \pm}(x)= & \left(4 N_{\mathrm{SP}}\right)^{-2} \mid \int_{-\infty}^{\infty}\left[\mathrm{E}_{\mathrm{z}, \mathrm{SP}}(z) \mathrm{H}_{\mathrm{y}}(x, z)\right. \\
& \left. \pm \mathrm{E}_{\mathrm{z}}(x, z) \mathrm{H}_{\mathrm{y}, \mathrm{SP}}(z)\right]\left.\mathrm{d} z\right|^{2},
\end{aligned}
$$

for $x \in[f a / 2, a-f a / 2]$ modulo $a, f=w / a$ being the fill factor. In Eq. (1), $\quad N_{\mathrm{SP}}=1 / 2 \int \mathrm{E}_{\mathrm{z}, \mathrm{SP}}(z) \mathrm{H}_{\mathrm{y}, \mathrm{SP}}(z) \mathrm{d} z$

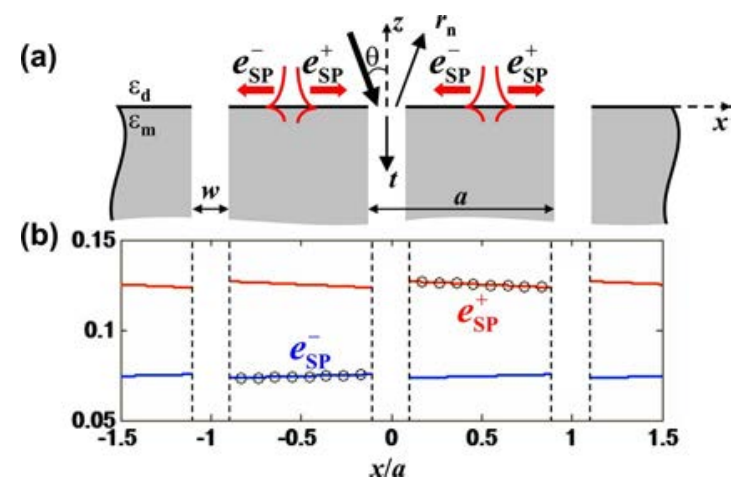

FIG. 1. (Color online) (a) Interface $(z=0)$ between a homogeneous medium (permittivity $\varepsilon_{\mathrm{d}}=n_{\mathrm{d}}^{2}$ ) and a periodic half-space composed of an array of semi-infinite slits perforated in the metal (permittivity $\varepsilon_{\mathrm{m}}$ ). The $r_{\mathrm{n}} \mathrm{s}$ are the plane-wave scattering coefficients used in the analytical model, and $e_{\mathrm{SP}}^{+}$and $e_{\mathrm{SP}}^{-}$represent the normalized SPP-excitation rates. (b) $e_{\mathrm{SP}}^{+}$(red) and $e_{\mathrm{SP}}^{-}$ (blue) values calculated with the fully-vectorial RCWA formalism for gold $\left(\varepsilon_{\mathrm{m}}=-9.04+1.06 \mathrm{i}\right), \lambda=a=0.7 \mu \mathrm{m}, w=100 \mathrm{~nm}$, and $\theta=10^{\circ}$. The black circles show the SPP damping, $\exp \left[-2 \operatorname{Im}\left(k_{\mathrm{SP}}\right)|x|\right]$, on flat metal surfaces. 


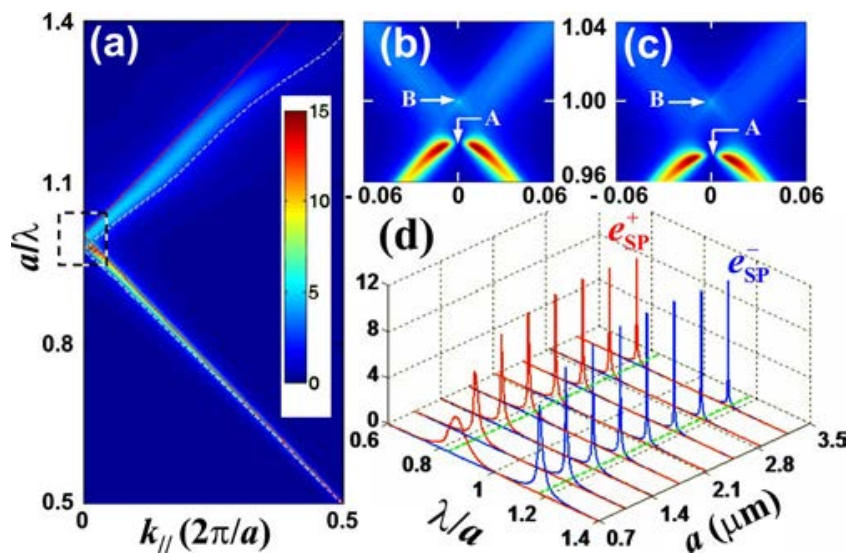

FIG. 2. (Color online) (a) Total SPP-excitation rates $e_{\mathrm{SP}}^{+}+e_{\mathrm{SP}}^{-}$in the $\left(\omega-k_{\|}\right)$ diagram at visible and infrared frequencies. The results hold for the geometry of Fig. $1 \quad(a=0.7 \mu \mathrm{m}$ and $w=100 \mathrm{~nm})$ and are obtained with the RCWA formalism. The solid-red and dashed-white curves, respectively, represent the Rayleigh anomalies and the folded dispersion relation of SPPs on flat gold surfaces. (b) Enlarged views in the vicinity of the $\Gamma$ point, $k_{\|}=0$ and $a=\lambda$ [shown in the dashed box in (a)]. The points A and B represent the SPP and Rayleigh wavelength at normal incidence. (c) The same as in (b) but calculated with the analytical model. (d) $e_{\mathrm{SP}}^{+}$(red) and $e_{\mathrm{SP}}^{-}$(blue) values obtained with the analytical model of Eq. (2) for various grating periods, and for $\theta=10^{\circ}$ and $w / a=1 / 7$. The dashed-green lines locate the Rayleigh wavelengths.

$\left[\approx\left|\varepsilon_{\mathrm{m}}\right|^{1 / 2} /\left(4 \omega \varepsilon_{0} \varepsilon_{\mathrm{d}}^{3 / 2}\right)\right]$ and the plus or minus superscripts hold for SPPs propagating in the positive or negative $x$-directions. Considering that the amplitude of the incident plane wave is unitary, $e_{\mathrm{SP}}^{ \pm}$actually represent the ratios between the magnetic-field of the launched SPPs and that of the incident plane-waves; it could be referred to as the normalized SPP-excitation rates. Field enhancements correspond to rates that are larger than one. Once the scattered field is calculated with the rigorous coupled wave analysis (RCWA), ${ }^{16}$ the integral in Eq. (1) is obtained by using classical numerical integration methods. Figure 1(b) shows typical $e_{\mathrm{SP}}^{ \pm}$profiles obtained at visible frequencies. From Eq. (1), it is easily derived that $e_{\mathrm{SP}}^{+}$and $e_{\mathrm{SP}}^{-}$are pseudoperiodic functions of $x$. As expected, $e_{\mathrm{SP}}^{+}$decays on every flat part of the ridges, with a decaying rate strictly equal to the exponential damping $\exp \left[-2 \operatorname{Im}\left(\mathrm{k}_{\mathrm{SP}}\right) x\right]$ of SPPs on flat metallic surfaces (black circles). The asymmetry of $e_{\mathrm{SP}}^{+}$and $e_{\mathrm{SP}}^{-}$is due to the oblique illumination $\left(\theta=10^{\circ}\right)$.

Because the SPP damping over a single ridge is very weak [it is only $3 \%$ at visible frequencies, see Fig. 1(b)], we may simply consider the averaged value of $e_{\mathrm{SP}}^{ \pm}(x)$ along every ridge, a value denoted by $e_{\mathrm{SP}}^{ \pm}$hereafter. Figure 2(a) shows $e_{\mathrm{SP}}^{+}+e_{\mathrm{SP}}^{-}$in the $\left(\omega-k_{\|}\right)$diagram, $k_{\|}=k_{0} \mathrm{n}_{\mathrm{d}} \sin (\theta)$. The results are obtained with the RCWA for the gold geometry of Fig. 1(a). Several important properties are derived. First, we note that, all in all, the excitation rate is very weak. On average in the $\left(\omega-k_{\|}\right)$plane, it is only a few percents [we find $\left\langle e_{\mathrm{SP}}^{ \pm}\right\rangle$ $<0.05$ for this specific geometry]. Second, the excitation rate exceeds one only in some narrow bands that are approximately delimited by two following sets of curves: the Rayleigh anomalies defined by the passing-off of a diffraction order $\left[2 \pi / \lambda_{\mathrm{R}} \sin (\theta)+2 m \pi / a= \pm k_{0}\right.$ shown with the solid-red curves] and the folded dispersion relations of SPPs on flat metallic surfaces $\left[2 \pi / \lambda_{\mathrm{SP}} \sin (\theta)+2 m \pi / a= \pm k_{\mathrm{SP}}\right.$ shown with the dashed-white curves], $\lambda_{\mathrm{R}}$ and $\lambda_{\mathrm{SP}}$ being the Rayleigh and SPP wavelengths. Third, the normalized SPP- excitation rate is not overwhelmingly strong (the averaged peak-enhancement in the bright bands is only four). This is because a significant fraction of launched SPPs is drained off at every slit, preventing the formation of a strong collective SPP resonance at the interface. Finally, we note that, in the vicinity of the point $A$ on the lower branch [see Fig. 2(b)], the resonance is the most acute $\left(e_{\mathrm{SP}}^{ \pm} \approx 15\right)$. This strong enhancement has been interpreted as resulting from a collective antisymmetric resonance formed by two counter-propagating delocalized SPPs with almost opposite amplitudes. ${ }^{17}$ For normal incidence, the mode becomes dark (point A), and the maximum SPP excitation exactly occurs at the Rayleigh anomaly (point B).

In order to obtain useful expressions for the excitation rate, we now develop an analytical model. The electromagnetic field in the slits is assumed to be solely composed of the fundamental Bloch mode of the slit array. For the sake of accuracy, we use surface impedance $\left(Z_{\mathrm{s}}=\varepsilon_{\mathrm{m}}^{-1 / 2}\right)$ boundary conditions $^{18}$ for matching the tangential field components at the interface. Although the scattered field in the uniform dielectric half-space above the slit array stems from an infinite Rayleigh series of reflected plane-waves with amplitude coefficients $r_{\mathrm{n}}$, we have analytically shown that only a single reflection coefficient $\left(r_{ \pm 1}\right)$ significantly contributes to the excitation rate. Under those considerations and for the two branches of Fig. 2(a), Eq. (1) leads to

$$
e_{\mathrm{SP}}^{ \pm}=\left|\frac{2\left(Z_{\mathrm{S}}-n_{\mathrm{eff}}\right) f g_{0} g_{ \pm 1} \gamma_{0}}{\left(\gamma_{0}+Z_{\mathrm{S}}\right)\left(\gamma_{ \pm 1}+Z_{\mathrm{S}}\right)(1-I)}\right|^{2}\left|\frac{\gamma_{\mathrm{SP}}\left(\alpha_{ \pm 1} \pm k_{\mathrm{SP}}\right)}{k_{\mathrm{SP}}\left(\gamma_{ \pm 1} k_{0}+\gamma_{\mathrm{SP}}\right)}\right|^{2},
$$

where the first factor is equal to $\left|r_{ \pm 1}\right|^{2}, \quad I=\Sigma_{\mathrm{m}}\left[\left(Z_{\mathrm{s}}\right.\right.$ $\left.\left.-n_{\mathrm{eff}}\right) f g_{\mathrm{m}}^{2} /\left(\gamma_{\mathrm{m}}+Z_{\mathrm{s}}\right)\right], \quad g_{\mathrm{m}}=1 / w \int_{-w / 2}^{w / 2} \varphi(x) \exp \left(\mathrm{j} \alpha_{\mathrm{m}} x\right) \mathrm{d} x, \quad$ with $\alpha_{\mathrm{m}}=k_{\|}+2 m \pi / a, \gamma_{\mathrm{m}}^{2} k_{0}^{2}+\alpha_{\mathrm{m}}^{2}=\varepsilon_{\mathrm{d}} k_{0}^{2}$, and $n_{\text {eff }}$ denoting the effective index of the fundamental slit-array mode $\varphi(x)$. The predictions of the analytical model are shown in Fig. 2(c) in the vicinity of $\mathrm{A}$ and $\mathrm{B}$ in the $\left(\omega-k_{\|}\right)$diagram. They quantitatively agree with the fully-vectorial RCWA computational results of Fig. 2(b), and can thus be used with confidence for further analysis.

Figure 2(d) shows the excitation rate for various grating periods covering visible and infrared illuminations. The results are obtained with the analytical model for $n=1$ (black curve) or -1 (blue curve) and for $\theta=10^{\circ}$. Importantly, we note that the maxima of the resonance peaks are almost independent of the periodicity $a$, but that the normalized peak widths, $\Delta \lambda / a$, rapidly shrink as $a$ increases. These trends are general. They also hold at point $\mathrm{B}$ for $\theta=0$, for which the closed-form expression in Eq. (2) considerably simplifies and reads as

$$
e_{\mathrm{SP}}^{+}=e_{\mathrm{SP}}^{-}=\left|\frac{4 f \operatorname{sinc}(\pi f)}{Z_{\mathrm{S}}+2 f \operatorname{sinc}^{2}(\pi f)}\right|^{2},
$$

for the peak maximum. A similar expression for the normalized peak width is easily derived

$$
\Delta \lambda / a \approx(2-\sqrt{2})\left|Z_{\mathrm{s}}\right|^{2} .
$$

Note that these equations are derived by assuming that $\left|Z_{\mathrm{S}}\right|$ $=\left|\varepsilon_{\mathrm{m}}\right|^{-1 / 2} \ll f$ a reasonable approximation for noble metals in the red and infrared bands. The peak maxima obtained at point B with Eq. (3) are shown in Fig. 3 with the solid-red 


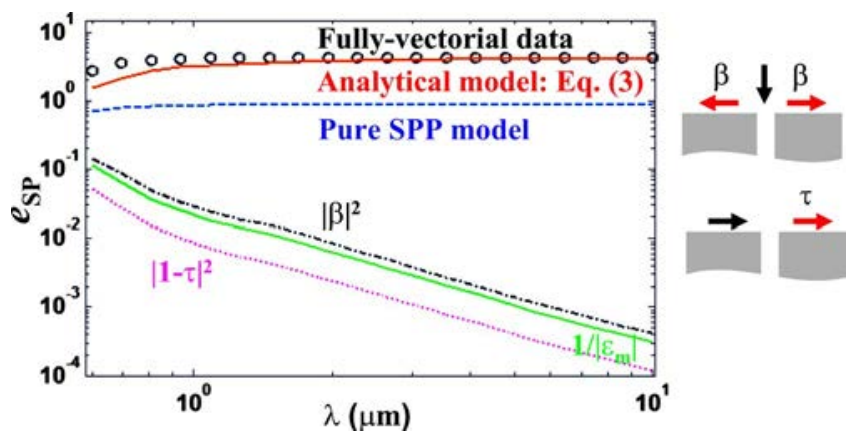

FIG. 3. (Color online) Microscopic interpretation for the influence of the metal permittivity on the normalized SPP-excitation rate for $\theta=0$. The two lower curves represents fundamental scattering coefficients, $\beta$ and $\tau$, for isolated slits whose widths are equal to those used for the grating calculation, $w=0.143 \lambda$. Both $|\beta|^{2}$ and $|1-\tau|^{2}$ scales as $\left|\varepsilon_{\mathrm{m}}\right|^{-1}$, shown with the solidgreen curve. The pure SPP model $\left(|\beta|^{2} /\left[4|1-\tau|^{2}\right]\right)$ and the analytical model predictions are shown, respectively, with dashed-blue and solid-red curves. The black circles show fully-vectorial RCWA data.

curve. They are well approximating the fully-vectorial RCWA data (circles).

It is well known that the SPP-excitation rate of isolated slits rapidly vanishes as $\left|\varepsilon_{\mathrm{m}}\right|^{-1}$ as one increases the wavelength by scaling all geometrical dimensions. ${ }^{11}$ Naively, one may expect that a similar behavior occurs for slit arrays. However that is not the case. In order to explain why, we refer to a microscopic model initially developed for analyzing the extraordinary optical transmission. The model ${ }^{19}$ assumes that solely the SPPs are responsible for the electromagnetic interaction between the slits in the array, and thus neglects the impact of quasi-CWs. For $\theta=0$, the peak maximum is approximately given by $|\beta|^{2} /\left[4|1-\tau|^{2}\right],{ }^{13,19,20}$ where $\tau$ is the SPP transmittance coefficient of a single slit (dotpurple curve in Fig. 3), and $\beta$ is the SPP-excitation coefficient by a single slit illuminated by a normally-incident plane wave, (dashed-dotted curve in Fig. 3). Since both $|1-\tau|^{2}$ and $|\beta|^{2}$ scales as $\left|\varepsilon_{\mathrm{m}}\right|^{-1}$, the pure SPP model (dashed-blue curve) also predicts that the excitation rate remains constant as the metal permittivity varies. The physical meaning is now clear: as one progressively move to lower frequencies, fewer SPPs are launched at every individual slit $\left(|\beta|^{2} \propto\left|\varepsilon_{\mathrm{m}}\right|^{-1}\right)$, but once launched, fewer SPPs are scattered by the adjacent slits (the SPP scattered in the forward direction $|1-\tau| \propto\left|\varepsilon_{\mathrm{m}}\right|^{-1 / 2}$ ). As a net effect, the SPP excitation on every individual ridge results from a perfect balance between a reduced local excitation rate and a longer collection (propagation) length $\left[\mathrm{L}_{\mathrm{SP}}\right.$ $\approx-a(2 \ln |\tau|)^{-1}$, if one neglects SPP back-reflections]. This statement is consistent with the decrease in the resonant width, either angularly or spectrally in agreement with Fig. 2(d) and Eq. (4). Although it provides the correct scaling law, the pure SPP model is only qualitative, as shown by the significant difference between the solid-red and dashed-blue curves in Fig. 3. Indeed, full assessment requires considering the quasi-CWs that are launched by the individual slits and their further cross-conversion to SPPs through scattering by adjacent slits. ${ }^{13}$ This two-step process, the dominant one even at near-infrared frequencies (Fig. 3), relies on scaling laws that are similar to those discussed in the pure SPP model, with a cross-conversion efficiency that decreases as the wavelength increases ${ }^{13}$ and an overall effect that is independent of the wavelength, as evidenced by the nearly constant difference between the dashed-blue and solid-red curves at long wavelengths.

In conclusion, we have studied the SPP-excitations rate on individual ridges of metallic gratings. We have provided fully-vectorial calculations, derived closed-form expressions and proposed a model that explains the main grating properties. The approach can be generalized to other geometries like groove arrays, hole arrays or non-periodic geometries with a finite number of indentations. We believe that the present analysis that fuses macroscopic and microscopic interpretations provides a helpful insight into a classical diffraction problem, and that the acquired comprehension may help designing plasmonic devices.

${ }^{1}$ R. W. Wood, Philos. Mag. 4, 396 (1902).

${ }^{2}$ R. Petit, Electromagnetic theory of gratings (Spinger, Berlin, 1980).

${ }^{3}$ T. W. Ebbesen, C. Genet, and S. I. Bozhevolnyi, Phys. Today 61, 44 (2008).

${ }^{4}$ P. Lalanne and J. P. Hugonin, Nat. Phys. 2, 551 (2006).

${ }^{5}$ W. Dai and C. Soukoulis, Phys. Rev. B 80, 155407 (2009).

${ }^{6}$ P. Lalanne, J. P. Hugonin, H. T. Liu, and B. Wang, Surf. Sci. Rep. 64, 453 (2009).

${ }^{7}$ Y. S. Jung, J. Wuenschell, T. Schmidt, and H. K. Kim, Appl. Phys. Lett. 92, 023104 (2008).

${ }^{8}$ B. Wang, L. Aigouy, E. Bourhis, J. Gierak, J. P. Hugonin, and P. Lalanne, Appl. Phys. Lett. 94, 011114 (2009).

${ }^{9}$ H. W. Kihm, J. H. Kang, J. S. Kyoung, K. G. Lee, M. A. Seo, and K. J. Ahn, Appl. Phys. Lett. 94, 141102 (2009).

${ }^{10} \mathrm{P}$. Lalanne, J. P. Hugonin, and J. C. Rodier, J. Opt. Soc. Am. A Opt. Image Sci. Vis 23, 1608 (2006).

${ }^{11}$ H. F. Schouten, N. Kuzmin, G. Dubois, T. D. Visser, G. Gbur, P. F. A. Alkemade, H. Blok, G. W. 't Hooft, D. Lenstra, and E. R. Eliel, Phys. Rev. Lett. 94, 053901 (2005).

${ }^{12}$ G. Gay, O. Alloschery, B. Viaris de Lesegno, C. O'Dwyer, J. Weiner, and H. J. Lezec, Nat. Phys. 2, 262 (2006).

${ }^{13}$ X. Y. Yang, H. T. Liu, and P. Lalanne, Phys. Rev. Lett. 102, 153903 (2009).

${ }^{14}$ F. López-Tejeira, S. G. Rodrigo, L. Martín-Moreno, F. J. García-Vidal, E. Devaux, T. W. Ebbesen, J. R. Krenn, I. P. Radko, S. I. Bozhevolnyi, M. U. González, J. C. Weeber, and A. Dereux, Nat. Phys. 3, 324 (2007).

${ }^{15}$ E. D. Palik, Handbook of Optical Constants of Solids (Academic, New York, 1985)

${ }^{16}$ M. G. Moharam, E. B. Grann, D. A. Pommet, and T. K. Gaylord, J. Opt. Soc. Am. A Opt. Image Sci. Vis 12, 1068 (1995).

${ }^{17}$ P. Lalanne, C. Sauvan, J. P. Hugonin, J. C. Rodier, and P. Chavel, Phys Rev. B 68, 125404 (2003).

${ }^{18}$ H. Lochbihler, Phys. Rev. B 50, 4795 (1994).

${ }^{19}$ H. Liu and P. Lalanne, Nature (London) 452, 728 (2008)

${ }^{20}$ J. S. Q. Liu, J. S. White, S. Fan, and M. L. Brongersma, Opt. Express 17, 17837 (2009). 